\title{
Evaluation of a fluorescent monoclonal antibody reagent for identification of cultured Neisseria gonorrhoeae
}

\author{
C. A. ISON, A. TANNA and C. S. F. EASMON
}

Department of Medical Microbiology, Wright Fleming Institute, St Mary's Hospital Medical School, London W2 1PG

\begin{abstract}
Summary. We evaluated a new fluorescent monoclonal antibody reagent for confirmation of identity of Neisseria gonorrhoeae. The reagent correctly identified all 161 fresh clinical isolates of $N$. gonorrhoeae, which included 11 penicillinase producing strains (PPNG). The reagent also correctly identified 21 stored PPNG strains. No cross reactions were seen with 58 fresh clinical isolates of $N$. meningitidis, 12 stored strains of $N$. lactamica, or with strains of Gardnerella vaginalis, lactobacilli, Candida spp., Staphylococcus epidermidis or Enterobacteriaceae. Some cross reaction was noted with strains of $S$. aureus, probably related to cell-wall protein A. However, this reagent was highly sensitive and specific for use against oxidase positive, gramnegative cocci isolated in London.
\end{abstract}

\section{Introduction}

Identification of Neisseria gonorrhoeae in a clinical laboratory must be rapid and accurate, and may be of medico-legal as well as clinical significance. In the United Kingdom it is most commonly achieved by conventional carbohydrate-utilisation tests which require pure cultures and at least overnight incubation. Alternative methods include rapid carbohydrate-degradation tests based on the principle described by Kellogg and Turner (1973), enzymic tests with chromogenic substrates (Janda et al., 1985), or immunological methods (Young and Reid, 1984). All these rapid techniques produce a result within $4 \mathrm{~h}$ of isolation of the presumptive neisseria.

Two types of immunological method are currently available, coagglutination and direct fluorescent staining of the organism. The sensitivity of coagglutination by a monoclonal reagent at 95$100 \%$ is better than that of the polyclonal antibody system originally used (Barnham and Glynn, 1978; Philip et al., 1984; Young and Reid, 1984). However, commercially available fluorescent antibody reagents, usually containing polyclonal antibody, have suffered from a lack of both sensitivity and specificity (Freundlich et al., 1982). We have evaluated a new fluorescent-antibody (FA) reagent

Received 31 July 1987; accepted 2 Oct. 1987.
(Syva Co., Palo Alto, CA, USA) containing monoclonal IgG antibodies.

\section{Material and methods}

\section{Strains}

A total of 161 isolates of $N$. gonorrhoeae (including 11 PPNG strains) and 58 isolates of $N$. meningitidis was obtained from primary cultures from patients attending the Praed Street Clinic, London. The source of the isolates is shown in table I. A further 21 isolates of PPNG and 12 isolates of $N$. lactamica from our collection of cultures stored in liquid nitrogen were also tested.

A range of bacterial isolates (numbers tested in brackets) including Gardnerella vaginalis (10), lactobacilli (1), Staphylococcus epidermidis (5), S. aureus (4), Candida spp. (3), Escherichia coli (5), Klebsiella oxytoca (1), K. pneumoniae (1), Proteus vulgaris (1), Enterobacter agglomerans (1) and Acinetobacter sp. (1) was tested to evaluate the specificity of the reagent.

\section{Culture}

All specimens from patients attending the clinic were inoculated directly onto neisseria isolation medium containing $\mathrm{GC}$ agar base (BBL) $36 \mathrm{~g} / \mathrm{L}$, IsoVitalex (BBL) $1 \%$, vancomycin $3 \mu \mathrm{g} / \mathrm{ml}$, colistin 100 units $/ \mathrm{ml}$, trimethoprim $5 \mu \mathrm{g} / \mathrm{ml}$, and amphotericin $1.5 \mu \mathrm{g} / \mathrm{ml}$. After inoculation, plates were incubated at $36^{\circ} \mathrm{C}$ in an atmosphere of $\mathrm{CO}_{2} 7 \%$ in air until transported to the laboratory, where they were re-incubated for up to $48 \mathrm{~h}$. Stored isolates of 
Table I. Source of primary isolates of Neisseria spp.

\begin{tabular}{|c|c|c|c|c|c|c|c|c|c|}
\hline \multirow[b]{4}{*}{ Organism } & \multicolumn{9}{|c|}{ Number of isolates tested from } \\
\hline & \multirow[t]{3}{*}{ Total } & \multicolumn{5}{|c|}{ Male patients } & \multicolumn{3}{|c|}{ Female patients } \\
\hline & & \multicolumn{2}{|c|}{ Heterosexual } & \multicolumn{3}{|c|}{ Homosexual } & \multirow[b]{2}{*}{$\mathbf{U}$} & \multirow[b]{2}{*}{$\mathrm{C}$} & \multirow[b]{2}{*}{$\mathrm{P}$} \\
\hline & & $\mathrm{U}$ & $P$ & $\mathrm{U}$ & $\mathbf{R}$ & $P$ & & & \\
\hline$N$. gonorrhoeae (non-PPNG) & 150 & 69 & 0 & 11 & 8 & 0 & 17 & 45 & 0 \\
\hline N. gonorrhoeae (PPNG) & 11 & 7 & 0 & 2 & 0 & 0 & 0 & 2 & 0 \\
\hline N. meningitidis & 58 & 0 & 11 & 0 & 2 & 36 & 0 & 0 & 9 \\
\hline
\end{tabular}

$\mathrm{U}=$ urethra, $\mathrm{P}=$ pharynx, $\mathrm{R}=$ rectum, $\mathrm{C}=$ cervix.

PPNG and $N$. lactamica were cultured on the above medium without the addition of antibiotics.

Isolates of $G$. vaginalis and lactobacilli were grown on $5 \%$ human-blood agar, staphylococci on $5 \%$ horse-blood agar, Candida spp. on Sabouraud's agar and the remaining isolates on MacConkey agar.

\section{Identification}

Neisseria spp. were identified as oxidase-positive, gramnegative cocci. $N$. gonorrhoeae was distinguished from other neisseriae by its ability to utilise only glucose. Carbohydrate-utilisation tests were performed on serumfree medium containing glucose, maltose or sucrose poured into petri dishes (Flynn and Waitkins, 1972). Penicillinase-producing strains were detected with a chromogenic cephalosporin (nitrocefin, Oxoid) (O'Callaghan et al., 1972). $N$. lactamica was distinguished from $N$. meningitidis by its ability to split ortho-nitrophenylgalactoside as previously described (Ison et al., 1984).

$G$. vaginalis isolates were identified as oxidase- and catalase- negative, gram-variable bacilli that were $\beta$ haemolytic on human- but not horse-blood agar. Staphylococci were identified as catalase-positive gram-positive cocci and distinguished by coagulase and DNAase activity. All gram-negative bacilli isolated on MacConkey agar were identified by API20E strips (API Laboratory Products Ltd).

\section{Immunofluorescence technique}

A thin smear of each type of colony was prepared by lightly touching five colonies and emulsifying in a $5-\mu 1$ drop of distilled water on a glass slide. The smears were allowed to dry completely in air and then gently heat fixed. The fluorescent-antibody reagent (Microtrak; Syva Co.) $30 \mu \mathrm{l}$, was added to each test smear, and to smears of known positive and negative control organisms. The slides were then incubated for $15 \mathrm{~min}$ at $37^{\circ} \mathrm{C}$ in a wellhumidified chamber to prevent drying which may lead to incorrect results. After incubation, excess reagent was removed by shaking and the slides rinsed for 5-10 $\mathrm{s}$ with a gentle stream of distilled water. The smears were then thoroughly dried in air and mounted in the solution provided. When possible, slides were read immediately or stored at $2-8^{\circ} \mathrm{C}$ in the dark and read within $24 \mathrm{~h}$.

The degree of fluorescence was determined with a Laborlux microscope (Leitz Instruments) and a $100 \times$ oilimmersion objective. Each smear was graded: $0=$ no fluorescence; $1+=$ all cooci were green and fluoresced at a low intensity; $2+=$ all cocci fluoresced, with clearly visible rims, medium intensity; $3+=$ all cocci fluoresced brightly with very clear rims; $4+=$ all cocci fluoresced very brightly.

\section{Results}

From 219 primary cultures containing oxidasepositive gram-negative cocci, 161 strains of $N$. gonorrhoeae (including $11 \mathrm{PPNG}$ ) and 58 strains of $N$. meningitidis were correctly identified by the FA reagent. The fluorescent reactions of these isolates together with the 21 stored strains of $N$. gonorrhoeae and 11 of $N$. lactamica are shown in table II. All 182 strains of $N$. gonorrhoeae gave positive fluores-

Table II. Results of fluorescent-antibody reagent tests with various Neisseria spp.

\begin{tabular}{|c|c|c|c|c|c|c|}
\hline \multirow{2}{*}{ Organism } & \multirow{2}{*}{$\begin{array}{c}\text { Total } \\
\text { number } \\
\text { tested }\end{array}$} & \multicolumn{5}{|c|}{$\begin{array}{l}\text { Number that gave } \\
\text { fluorescence intensity }\end{array}$} \\
\hline & & 0 & $1+$ & $2+$ & $3+$ & $4+$ \\
\hline \multicolumn{7}{|l|}{ Primary isolates } \\
\hline $\begin{array}{l}N . \text { gonorrhoeae } \\
\text { (non-PPNG) }\end{array}$ & 150 & 0 & 6 & 31 & 49 & 64 \\
\hline $\begin{array}{l}N . \text { gonorrhoeae } \\
\text { (PPNG) }\end{array}$ & 11 & 0 & 0 & 4 & 2 & 5 \\
\hline N. meningitidis & 58 & 58 & 0 & 0 & 0 & 0 \\
\hline \multicolumn{7}{|l|}{ Stock isolates } \\
\hline $\begin{array}{l}\text { N. gonorrhoeae } \\
\text { (PPNG) }\end{array}$ & 21 & 0 & 2 & 8 & 5 & 6 \\
\hline N. lactamica & 12 & 12 & 0 & 0 & 0 & 0 \\
\hline
\end{tabular}


cent reactions, all but eight of which were $\geqslant 2+$ intensity. The age of the culture did not affect this intensity. None of the strains of $N$. meningitidis or $N$. lactamica reacted with the fluorescent-antibody reagent.

Of all the other species tested only three strains of $S$. aureus showed any fluorescence. This was seen in only a minority of cocci, and these staphylococci did not resemble typical gonococci morphologically. No attempt was made to block this reaction with serum of any kind as this commercial reagent is intended for use solely with oxidase-positive gram-negative cocci.

\section{Discussion}

The FA reagent correctly identified all the isolates of $N$. gonorrhoeae from primary cultures plates. The test was simple and quick to perform and gave easily readable results. It was used successfully on colonies without subculture and was not affected by the viability or age of the culture. The isolates of PPNG tested in this study all gave a positive result. Immunologically-based gonococcal reagents have been reported to fail to identify some isolates of PPNG (Waitkins and Anderson, 1982; Philip et al., 1984). In our experience, some strains of PPNG

\section{REFERENCES}

Barnham M, Glynn A A 1978 Identification of clinical isolates of Neisseria gonorrhoeae by a coagglutination test. Journal of Clinical Pathology 31 : 189-193.

Flynn J, Waitkins S A 1972 A serum free medium for testing fermentation reactions in Neisseria gonorrhoeae. Journal of Clinical Pathology 25 : 525-527.

Freundlich L F, Rosenthal S L, Hochberg F P, Trogele M R 1982 Comparison of methods for the immunological identification of Neisseria gonorrhoeae in clinical specimens using commercially-obtained reagents. American Journal of Clinical Pathology 77: 456-458.

Ison C A, Bellinger C M, Glynn A A 1984 Plasmids in throat and genital isolates of meningococci. Journal of Clinical Pathology 37: 1123-1 128.

Janda W M, Ulanday M G, Bohnoff M, LeBeau L J 1985 Evaluation of the RIM-N, Gonochek II, and Phadebact systems for the identification of pathogenic Neisseria spp. are slow to utilise carbohydrates, particularly after only one or two subcultures. This FA reagent offers a useful alternative which would enable quick identification and reporting of a penicillin-resistant isolate.

We did not detect any cross-reactivity with other species of Neisseria tested. In our laboratory $N$. meningitidis and $N$. lactamica, but not other neisseriae are commonly isolated from pharyngeal cultures. When used against oxidase-positive, gramnegative cocci isolated on our neisseria selective medium, the reagent was $100 \%$ specific.

Spurious staining was only observed with isolates of $S$. aureus. This was probably due to protein A on these strains which binds IgG non-specifically, resulting in positive fluorescence. This problem is encountered with any reagent that uses $\mathrm{IgG}$ antibody and it does not present a practical problem in a clinical laboratory where only oxidase-positive, gram-negative cocci are tested.

This immunofluorescence test is particularly useful because it requires neither viable organisms nor the overnight incubation of subcultures which is frequently necessary for carbohydrate-utilisation tests of any type. Its sensitivity, specificity and ease of use make it an attractive alternative to other rapid tests.

and Branhamella catarrhalis. Journal of Clinical Microbiology 21: 734-737.

Kellogg D S, Turner E M 1973 Rapid fermentation confirmation of Neisseria gonorrhoeae. Applied Microbiology 25: 550-552.

O'Callaghan C H, Morris A, Kirby S M, Shingler, A H 1972 Novel method for detection of beta-lactamases by using a chromogenic cephalosporin substrate. Antimicrobial Agents and Chemotherapy $1: 283-288$.

Philip S K, Ison C A, Easmon C S F 1984 Coagglutination identification of Neisseria gonorrhoeae. British Journal of Venereal Diseases 60 : 66.

Young H, Reid K G 1984 Immunological identification of Neisseria gonorrhoeae with monoclonal and polyclonal antibody coagglutination reagents. Journal of Clinical Pathology 37: 1276-1281.

Waitkins S A, Anderson R D 1982 Failure of the fluorescent antibody reaction to identify penicillinase-producing gonococci. Journal of Clinical Pathology 35: 215-218. 
